\title{
Long-Term Outcome in Transient Global Amnesia Patients with and without Focal Hyperintensities in the CA1 Region of the Hippocampus
}

\author{
Ingo Uttner ${ }^{\mathrm{a}}$ Sascha Prexl ${ }^{\mathrm{a}}$ Wolfgang Freund ${ }^{\mathrm{b}}$ Alexander Unrath ${ }^{\mathrm{a}}$ \\ Dietmar Bengel $^{c}$ Roman Huber ${ }^{a}$ \\ Departments of a Neurology and ${ }^{\mathrm{b}}$ Diagnostic and Interventional Radiology, University of Ulm, Ulm, and \\ 'Department of Neurology, Krankenhaus St. Elisabeth, Ravensburg, Germany
}

\section{Key Words}

Hippocampus $\cdot$ DWI lesions $\cdot$ MRI $\cdot$ Amnesia $\cdot$ Transient

global amnesia

\begin{abstract}
Focal hippocampal diffusion-weighted imaging (DWI) lesion patterns are detected in transient global amnesia (TGA) patients in different frequency. It has been speculated that acute diffusion restrictions are associated with a worse outcome. Therefore, we evaluated the influence of acute DWI lesions on the cognitive long-term outcome in TGA patients. Seventeen otherwise healthy patients with the clinical syndrome of TGA, who had MRI investigations on admission as well as 1 day later, were investigated with a comprehensive neuropsychological test battery 2 years later. Acute hippocampal DWI lesions in TGA patients were detected in almost two thirds of the patients. Psychometric evaluation revealed no differences in cognitive performance between patients with and without DWI lesions as well as compared to healthy subjects. In addition, no relapse of the attack has been recognized in either group of TGA patients.
\end{abstract}

Copyright $\odot 2012$ S. Karger AG, Basel
(C) 2012 S. Karger AG, Base

0014-3022/12/0673-0155\$38.00/0

Fax +41613061234 E-Mail karger@karger.ch www.karger.com

\section{Introduction}

Transient global amnesia (TGA) is a clinical condition characterized by sudden memory loss without further signs of cognitive impairment or concomitant focal neurological features. During the attack patients are conscious and alert, but show a severe anterograde amnesia which may extend also to variable retrograde parts of the episodic memory. Although clinical, psychometric and neuroimaging data suggest an explicit involvement of cerebral structures implicated in memory processing, the exact etiology of the disorder is still a matter of debate.

Proposed mechanisms include migraine, epilepsy and focal ischemia as well as venous congestion due to retrograde venous cerebral blood flow [1].

However, according to the overall low relapse rates of $10 \%$ for a second and of only $2-3 \%$ for a third or further episode, none of these pathophysiological theories seems to be convincing as these diseases show a substantially higher recurrence. Alternatively, stress-related causes have been discussed, as in more than $90 \%$ of the patients emotionally disturbing situations have been reported. It was also hypothesized that hippocampal sulcal cavities, residual cysts arising from a lack of vestigial hippocampal fissure obliteration during fetal development, contribute

PD Dr. I. Uttner

Department of Neurology

University of Ulm

Oberer Eselsberg 45, DE-89081 Ulm (Germany)

Tel.+49 731 1770, E-Mail ingo.uttner@uni-ulm.de 
Table 1. Demographic characteristics of the two TGA subgroups and the healthy controls

\begin{tabular}{llllll}
\hline Group & $\begin{array}{l}\text { Age } \\
(\text { median; } \\
\text { range) }\end{array}$ & Gender & $\begin{array}{l}\text { Years of } \\
\text { education }\end{array}$ & $\begin{array}{l}\text { Verbal intelligence } \begin{array}{l}\text { Months between TGA } \\
\text { (WST) } \\
\text { (median; range) }\end{array} \\
\text { nation (median; range) }\end{array}$ \\
\hline Patients without DWI changes $(\mathrm{n}=6)$ & $70 ; 61-82$ & $\begin{array}{l}\text { female: } 2 \\
\text { male: }\end{array}$ & $\begin{array}{c}8-9: 3 \\
10-13: 3\end{array}$ & $\begin{array}{l}97 ; 79-129 \\
24 ; 14-33\end{array}$ \\
\hline Patients with DWI changes $(\mathrm{n}=11 ; 3$ bilateral) & $68 ; 55-77$ & $\begin{array}{l}\text { female: } 9 \\
\text { male: } 2\end{array}$ & $\begin{array}{c}8-9: 8 \\
10-13: 3\end{array}$ & $95 ; 82-122$ & $30.5 ; 15-38$ \\
\hline Healthy controls $(\mathrm{n}=15)$ & $65 ; 47-77$ & $\begin{array}{l}\text { female: } 7 \\
\text { male: } 8\end{array}$ & $\begin{array}{c}8-9: 6 \\
10-13: 9\end{array}$ & $104 ; 90-133$ \\
\hline
\end{tabular}

to the pathophysiology of this enigmatic condition [2]. A central point of discussion concerns the question of whether the small focal hippocampal lesions which were seen in the diffusion-weighted imaging (DWI) in approximately $70 \%$ of the TGA patients $24-72 \mathrm{~h}$ after symptom onset [3-5] reflect a certain etiology. Although these lesions are generally unspecific they typically are detected in cases of ischemic stroke representing a zone of irreversible damage. Therefore, they should be associated with a higher probability of at least partially persistent cognitive impairment and of a detectable neuronal loss. Whereas numerous investigators have reported such long-lasting neuropsychological deficits [6-11], several studies devoted to imaging parameters [12], and especially the few dealing with the time course of the DWI changes, describe a complete remission of the hippocampal lesions without structural sequelae $[3,4]$. The important question, i.e. whether the cognitive long-term outcome of patients with DWI lesions differs from those who reveal no signal alterations in the magnetic resonance imaging (MRI), has not been addressed so far.

In this study, therefore, we compared the neuropsychological performance of well-defined TGA patients with and without initial DWI changes at a mean of 2 years after the attack (median 25; range 14-38 months).

\section{Subjects and Methods}

\section{Subjects}

Eleven patients with acute diffusion restrictions (DWI lesion group: 8 unilateral, 3 bilateral) and 6 patients without diffusion lesions (non-DWI lesion group) in the 1.5T MRI during or directly after the amnestic episode were investigated. All patients had been admitted to the Department of Neurology of the University of Ulm between 2005 and 2006 because of the occurrence of a single TGA attack. At the time of investigation, the median follow-up since the TGA was 30.5 months in the DWI lesion group and 24 months in the non-DWI lesion group (table 1). All patients fulfilled the criteria of TGA according to Caplan [13]: (a) acute onset anterograde amnesia, witnessed by an observer and resolved within $24 \mathrm{~h}$; (b) no accompanying focal neurological symptoms during the attack; (c) no recent head injury; (d) no epileptic features either in the past or during the attack; (e) intact consciousness and personal orientation, and (f) no other cognitive impairment other than anterograde amnesia.

Further exclusion criteria were: the presence of psychiatric diseases since the TGA (such as dementia), treatment with corticosteroids in the last 12 months and a history of drug or alcohol abuse which could interfere with the cognitive performance.

The control group consisted of 15 healthy subjects ( 7 women, 8 men; median age 65 years; range $47-77$ years). To achieve a balance concerning age, sex and nutritional factors as well as intellectual stimulation and social status, spouses if available or close relatives were chosen. The groups did not differ in terms of arteriosclerotic risk factors, such as arterial hypertension, hypercholesterolemia, smoking, obesity, and former cardiovascular events. All subjects gave written informed consent to their participation in the study. The study protocol was approved by the Ethics Committee of the University of Ulm.

\section{Neuroimaging}

Complete MRI investigations were performed on a 1.5 Tesla Siemens Symphony clinical routine scanner (Siemens, Erlangen, Germany) equipped with a 1-channel standard head coil. The MRI protocol encompassed the acquisition of $\mathrm{T}_{1^{-}}, \mathrm{T}_{2^{-}}, \mathrm{T}_{2}{ }^{*}$ - and fluid-attenuated inversion recovery (FLAIR)-weighted imaging as well as arterial time-of-flight magnetic resonance angiography and DWI. Three DWI sequences were acquired of which one was oriented axial in AC-PC (anterior commissure to posterior commissure) angulation and covered the whole brain with a slice thickness of $5 \mathrm{~mm}$, while the two others covered the hippocampal region of both hemispheres in axial and coronal orientation with a 3-mm slice thickness. The sequence parameters of these hippocampus-angulated DWI sequences were as follows: repetition time 3,500 ms, echo time $104 \mathrm{~ms}$, field of view $200 \mathrm{~mm}$, image matrix $128 \times 128$, slice thickness $3 \mathrm{~mm}, 19$ slices, 4 averages, bandwidth $1,184 \mathrm{~Hz} / \mathrm{px}$, EPI factor 128 , 3-scan Trace and a b value of $1,000 \mathrm{~s} / \mathrm{mm}^{2}$. Total acquisition time of the conducted protocol was about $18 \mathrm{~min}$. All patients were investigated by MRI at the time of admission as well as on the next day. 
Table 2. Neuropsychological test battery as performed in TGA patients and healthy controls reflecting the different cognitive domains with special emphasis on verbal and nonverbal memory functions

\begin{tabular}{|c|c|c|}
\hline Test, reference & Domain/assessed function & Task/recorded parameters \\
\hline Digit span [24] & \multirow[t]{3}{*}{ Verbal memory } & Number of digits forward/backward \\
\hline $\begin{array}{l}\text { California verbal learning test, } \\
\text { German adaption }[25]\end{array}$ & & $\begin{array}{l}\text { Encoding (sum; max: } 75 \text { ), 30/60 min delayed free recall of a 16-word } \\
\text { list presented for } 5 \text { trials }\end{array}$ \\
\hline Logical memory [24] & & Free recall of a story 60 min after presentation (max: 25) \\
\hline $\begin{array}{l}\text { Block span, }[24] \\
\text { Visual paired associates }[24]\end{array}$ & \multirow[t]{3}{*}{ Nonverbal memory } & $\begin{array}{l}\text { Number of correctly replicated figures forward/backward } \\
\text { Encoding, } 60 \text { min delayed recognition of } 6 \text { visually presented } \\
\text { symbols and colors (max: 6) }\end{array}$ \\
\hline Rey complex figure test [26] & & Free recall of a figure 40 min after presentation (max: 36 ) \\
\hline Famous faces test & & $\begin{array}{l}\text { A 15-portrait sample of well-known politicians ( } 5 \text { recent, } 5 \text { who } \\
\text { were active in the years 1970-1985, } 5 \text { who were active between } 1950 \\
\text { and 1970); subjects were asked for name, party membership and } \\
\text { political function (max: 45) }\end{array}$ \\
\hline $\begin{array}{l}\text { Controlled oral word association } \\
\text { test [27] }\end{array}$ & \multirow[t]{2}{*}{$\begin{array}{l}\text { Phonemic/categorical } \\
\text { word fluency }\end{array}$} & Number of words beginning with F, A, S in 3 min \\
\hline Word production task [28] & & Number of words associated with the category 'supermarket' in $1 \mathrm{~min}$ \\
\hline Tonic alertness [29] & \multirow[t]{2}{*}{ Processing speed } & \multirow{2}{*}{$\begin{array}{l}\text { Motor reaction time (ms) on a single visual stimulus with (phasic } \\
\text { condition)/without (tonic condition) preceding of an acoustic signal }\end{array}$} \\
\hline Phasic alertness [29] & & \\
\hline Divided attention [29] & Attentional capacity & $\begin{array}{l}\text { Motor reaction time (ms) on simultaneously presented visual and } \\
\text { acoustic stimuli }\end{array}$ \\
\hline Incompatibility [29] & $\begin{array}{l}\text { Selective attention; } \\
\text { interference }\end{array}$ & $\begin{array}{l}\text { Motor reaction time (ms) on left- or right-side-directed visual } \\
\text { stimuli, which interfere with the side of presentation }\end{array}$ \\
\hline
\end{tabular}

Neuropsychological Assessment

A comprehensive neuropsychological test battery consisting of paper-pencil and PC-supported tests (table 1) was given to both patients and controls in the same order and at the same time of day to minimize distortion by influences of circadian rhythm. Also, to detect subtle memory deficits, a broad range of functions including working, declarative and retrograde memory was assessed. In addition, attentional and executive functions as well as global intellectual capacity were examined.

\section{Data Analysis}

Due to the small sample size, nonparametric tests were used for group comparisons. Results were regarded as being significant if $\mathrm{p} \leq 0.05$.

\section{Results}

\section{Demographic Characteristics}

Demographic data of the TGA samples and the healthy controls are given in table 2. General intelligence as estimated by a vocabulary test was comparable $(\mathrm{p}=0.13$,
Kruskal-Wallis test), as well as years of education ( $\mathrm{p}=$ $0.56, \chi^{2}$ test), age ( $p=0.12$, Kruskal-Wallis test) and gen$\operatorname{der}\left(p=0.23, \chi^{2}\right.$-test). Patients with and without hippocampal DWI lesions did not differ in the time interval between TGA onset and follow-up investigation ( $\mathrm{p}=$ 0.078, Mann-Whitney U test).

\section{DWI Lesions}

A first MRI was performed immediately after the patients' admission to hospital. According to the history reported, the median time delay between the first clinical symptoms and the imaging was approximately 255 min (range 90-405 min). In all patients a second MRI was done on the following day. The median time delay to this imaging was $29.5 \mathrm{~h}$ (range 16.5-41 h) after the beginning of the episode. In the primary imaging 6 acute DWI lesions in 4 out of 17 patients (23.5\%) were detected. There was a slight difference in the time delay to imaging between both groups of DWI-positive and DWI-negative patients (300 vs. $237.5 \mathrm{~min}$, respectively). 


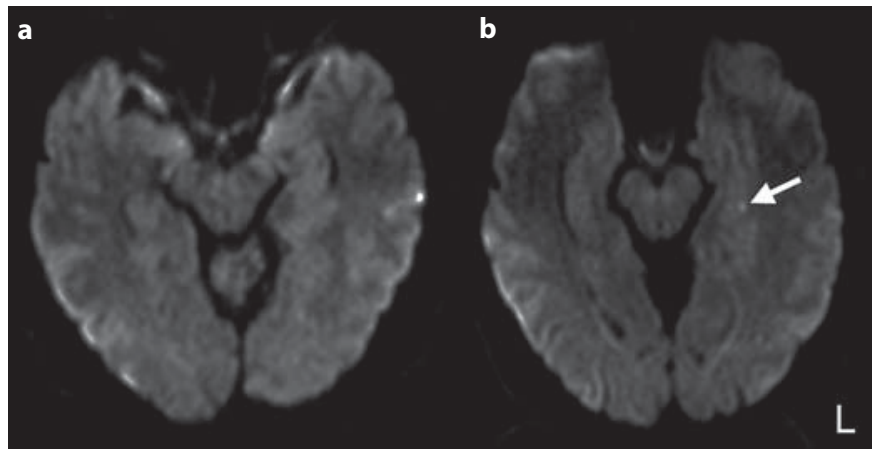

Fig. 1. Diffusion-weighted MRI at two different time points: $4 \mathrm{~h}$ (a) and $26 \mathrm{~h} \mathrm{(b)} \mathrm{after} \mathrm{onset} \mathrm{of} \mathrm{TGA} \mathrm{symptoms.} \mathrm{Image} \mathbf{b}$ shows a slight dot-like DWI restriction in the left dorsolateral hippocampal region. $\mathrm{L}=\mathrm{Left}$.

The proportion of DWI-positive patients increased to nearly two thirds (64.7\%) at the time of the second imaging, when 14 acute DWI lesions were detected in 11 out of 17 patients. Three patients demonstrated bilateral lesions, while in 8 patients unilateral diffusion restrictions were detected. All lesions were situated in the CA1 section of the hippocampus (fig. 1). There was a slight predominance of the left side, which was affected in $62.5 \%$ of cases.

\section{Cognitive Performance}

As shown in figure 2, patients and controls performed quite equally in the neuropsychological assessment. Significant group differences were observed only with respect to the Digit Span forward ( $\mathrm{p}=0.026$, Kruskal-Wallis test) and backward ( $\mathrm{p}=0.008$, Kruskal-Wallis test), and to the encoding section of the California verbal learning test ( $\mathrm{p}=0.003$, Kruskal-Wallis test). Subsequent single contrasts by means of the Mann-Whitney U test showed a significantly lower learning rate in the TGA patients without DWI lesions compared to those with DWI lesions $(\mathrm{p}=0.015)$ and the controls $(\mathrm{p}=0.001)$, whose test performances did not differ $(\mathrm{p}=0.087$, MannWhitney U test). Concerning the Digit Span forward and backward, controls scored significantly better than the patients with DWI lesions ( $p=0.005, p=0.004$, MannWhitney $U$ test). Performance of the patients without DWI lesions did not differ from that of the controls $(\mathrm{p}=$ $0.235, \mathrm{p}=0.055$, Mann-Whitney $\mathrm{U}$ test) or of the TGA patients with DWI lesions ( $\mathrm{p}=0.525, \mathrm{p}=0.462$, MannWhitney U test).

\section{Discussion}

Despite numerous attempts to uncover its pathophysiology, TGA remains a clinical syndrome of unknown etiology. A broad range of diseases has been considered to be the underlying cause of the amnestic episodes including epileptic seizures, ischemic stroke and migraine. The detection of acute DWI lesions in the hippocampal area by high-resolution MRI was primarily interpreted in favor of ischemic stroke [14]. However, further investigations did not support this idea $[3,15,16]$. In concordance with the literature in our investigation acute DWI lesions developed in about two thirds of the TGA patients after a time delay of more than $24 \mathrm{~h}$, while 6 out of 17 patients did not show any acute diffusion restrictions even at that late time point. Based on a multifactorial etiology these different imaging characteristics (DWI lesion and non-DWI lesion patients) might reflect different underlying pathologies leading to a clinically identical or at least very similar syndrome. However, our results do not suggest a crucial role for acute DWI lesions discriminating patients with pathophysiologically different diseases leading to neuropsychologically different long-term sequelae. According to our comprehensive test battery the patient groups differed in only one single test parameter - the California Verbal Learning Test learning rate, which was higher in the patients with DWI lesions. This is in contrast to the assumption that these patients are the ones who should have a greater risk of memory deficit. Although the interval between TGA onset and neuropsychological assessment did not significantly differ between both patient groups, patients without DWI lesions were investigated somewhat earlier (24 vs. 30.5 months). This time difference could possibly contribute to the better encoding performance in the group with DWI lesions [11, 17, 18].

Moreover, the highly concordant test performance of patients and controls underlines the benign character of TGA [19] and suggests that the disease does not lead to substantial cognitive restrictions independently of DWI lesions.

Interestingly, the pattern of patients with and without DWI lesions within one syndrome is very well known from transient ischemic attacks. It has been shown that transient ischemic attack patients with DWI lesions, classified as 'transient symptoms associated with infarctions' [20] seem to have a significantly higher risk of recurrent disease already in the short term. Recently, a similar phenomenon has been reported even for TGA patients, when patients suffering from a second TGA episode have been shown to have a higher frequency of acute DWI lesions 


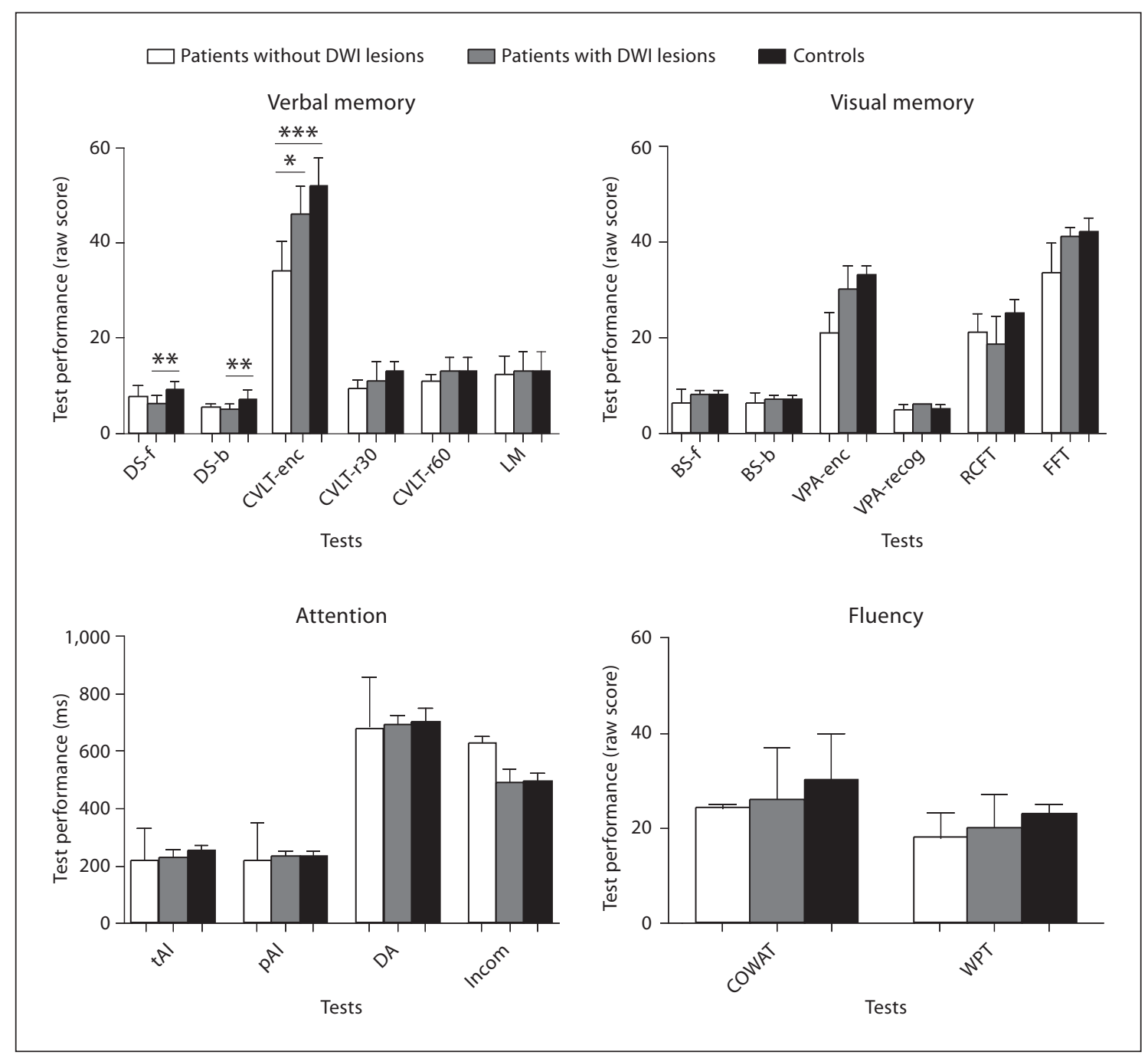

Fig. 2. Test performance (median and interquartile range) of the patients with $(n=11)$ and without $(n=6)$ DWI lesions in the hippocampus and of the controls $(n=15)$. DS- $\mathrm{f} /-\mathrm{b}=$ Digit Span forward/backward; CVLT-enc/ -r30/-r60 = California verbal learning test, encoding, 30/60 min delayed free recall; LM = logical memory, 60 min delayed recall; BS-f/-b = Block Span forward/backward; VPA-enc/-r60 = visual paired associates, encoding, 60 min delayed recognition; RCFT = Rey complex figure test, 40 min delayed recall; FFT = famous faces test, recall; $\mathrm{tAl} / \mathrm{pAl}=$ tonic/phasic alertness; $\mathrm{DA}=$ divided attention; $\mathrm{Incom}=$ incompatibility; $\mathrm{COWAT}=$ controlled oral word association test; WPT $=$ word production test.

[21]. However, in our cohort we did not see even one single relapse either in patients with or without acute DWI lesions over a time period of at least 2.5 years. Moreover, the frequency of DWI lesions in our cohort of first ever TGA patients was twice as high as in the report of Auyeung et al. [21], as we demonstrated, like several other groups $[1,14,22,23]$, acute DWI lesions in two thirds of patients. In addition, the clinical course without recurrence over a long time period does not argue for any impact of the DWI lesions on the long-term prognosis. Al- though we cannot exclude different etiologies leading to the different imaging patterns, we finally did not find differences in terms of neuropsychological outcome, prognosis and/or the risk of recurrent events according to the detection of DWI lesions in TGA patients.

\section{Disclosure Statement}

The authors report no conflict of interest. 


\section{References}

$\checkmark 1$ Bartsch T, Deuschl G: Transient amnesia: functional anatomy and clinical implications. Lancet Neurol 2010;9:205-214.

-2 Nakada T, Kwee IL, Fujii Y, Knight RT: Highfield, $\mathrm{T}_{2}$ reversed MRI of the hippocampus in transient global amnesia. Neurology 2005; 64:1170-1174

3 Bartsch T, Alfke K, Stingele R, et al: Selective affection of hippocampal CA-1 neurons in patients with transient global amnesia without long-term sequelae. Brain 2006;129: 2874-2884.

$\checkmark 4$ Ueno H, Naka H, Ohshita T, Wakabayashi S, Matsumoto M: Serial changes in delayed focal hippocampal lesions in patients with transient global amnesia. Hiroshima J Med Sci 2010;59:77-81.

$\checkmark 5$ Weon YC, Kim JH, Lee JS, Kim SY: Optimal diffusion-weighted imaging protocol for lesion detection in transient global amnesia. Am J Neuroradiol 2008;29:1324-1328.

-6 Mazzuchi A, Moretti G, Caffara P, Parma M: Neuropsychological functions in the followup of transient global amnesia. Brain 1980; 103:697-716.

7 Hodges JR, Oxbury SM: Persistent memory impairment following transient global amnesia. J Clin Exp Neuropsychol 1990;12:904920.

$\checkmark 8$ Cattaino G, Querin F, Pomes A, Piazza P: Transient global amnesia. Acta Neurol Scand 1984;70:385-390.

$\checkmark 9$ Borroni B, Agosti C, Brambilla C, Vergani V, Cottini E, Akkawi N, Padovani A: Is transient global amnesia a risk factor for amnestic mild cognitive impairment? J Neurol 2004;251:1125-1127.

-10 Caffarra P, Moretti G, Mazzuchi A, Parma M: Neuropsychological testing during a transient global amnesia episode and its follow-up. Acta Neurol Scand 1981;63:44-50.

- 11 LePira F, Giuffrida S, Macvi T, Reggio E, Zappala G, Perciavalle V: Cognitive findings after transient global amnesia: role of prefrontal cortex. Appl Neuropsychol 2005;12: 212-217.
12 Caffarra P, Concari L, Gardini S, Spaggiari S, Dieci F, Copelli S, Ghetti C, Venneri A Recovery from transient global amnesia following restoration of hippocampal and fronto-cingulate perfusion. Behav Neurol 2010; 22:131-139.

13 Caplan L: Transient global amnesia. Characteristic features and overview; in Markowitsch HJ (ed): Transient Global Amnesia and Related Disorders. Toronto, Hogrefe and Huber, 1990, pp 15-27.

14 Winbeck K, Etgen T, von Einsiedel HG, Röt tinger M, Sander D: DWI in transient global amnesia and TIA: proposal for an ischaemic origin of TGA. J Neurol Neurosurg Psychiatry 2005;76:438-441.

-15 Toledo M, Pujadas F, Grivé E, Alvarez-Sabin J, Quintana M, Rovira A: Lack of evidence for arterial ischemia in transient global amnesia. Stroke 2008;39:476-479.

16 Enzinger C, Thimary F, Kapeller P, et al: Transient global amnesia: diffusion-weighted imaging lesions and cerebrovascular disease. Stroke 2008;39:2219-2225.

17 Guillery-Girard B, Desgranges B, Urban C, Piolino P, de la Sayette V, Eustache F: The dynamic time course of memory recovery in transient global amnesia. J Neurol Neurosurg Psychiatry 2004;75:1532-1540.

18 Hodges JR, Ward CD: Observations during transient global amnesia. A behavioural and neuropsychological study of five cases. Brain 1989;112:595-620.

19 Uttner I, Weber S, Freund W, Schmitz B, Ramspott M, Huber R: Transient global amnesia - full recovery without persistent cognitive impairment. Eur Neurol 2007;58:146151.
20 Prabhakaran S, Chong JY, Sacco RL: Impact of abnormal diffusion-weighted imaging results on short-term outcome following transient ischemic attack. Arch Neurol 2007;64: 1105-1109.

21 Auyeung M, Tsoi TH, Cheung CM, Fong DY, Li R, Chan JK, Lau KY: Association of diffusion-weighted imaging abnormalities and recurrence in transient global amnesia. J Clin Neurosci 2011;18:531-534.

-22 Sedlaczek O, Hirsch JG, Grips E, Peters CN, Gass A, Woehrle J, Hennerici M: Detection of delayed focal MR changes in the lateral hippocampus in transient global amnesia. Neurology 2004;62:2165-2170.

23 Ryoo I, Kim JH, Kim S, Choi BS, Jung C, Hwang SI: Lesion detectability on diffusionweighted imaging in transient global amnesia: the influence of imaging timing and magnetic field strength. Neuroradiology 2011. [Epub ahead of print].

24 Härting C, Markowitsch HJ, Neufeld H, Calabrese P, Deisinger K, Kessler J: Deutsche Adaptation der revidierten Fassung der Wechsler Memory Scale. Bern, Huber, 1999.

25 Ilmberger J: Deutsche Version des California Verbal Learning Tests. München, Institut für Medizinische Psychologie der Universität München, 1988.

26 Meyers JE, Meyers KR: Rey Complex Figure Test and Recognition Trial. Professional Manual. Odessa, Psychological Assesment Resources Inc., 1995.

27 Benton AL, Hamsher K, Varney, NR, Spreen O: Contribution to Neuropsychological Assessment. New York, Oxford University Press, 1983

28 Kessler J, Denzler P, Markowitsch HJ: Demenztest: Eine Testbatterie zur Erfassung kognitiver Einschränkungen im Alter, ed 2, revised. Göttingen, Beltz Test GmbH, 1999.

29 Zimmermann P, Fimm B: Testbatterie zur Aufmerksamkeitsprüfung. Freiburg, PSYTEST, 1993. 